\title{
Routine and specialised techniques in the diagnosis of haematological neoplasms
}

\author{
B J Bain
}

\section{Introduction}

Haematological diagnosis has traditionally been based on light microscopy supplemented by cytochemistry. However, during the past decade the development of new techniques and increasingly effective and specific treatments have radically altered the approach of the haematologist to the diagnosis of haematological neoplasms. New methods of investigation, such as immunophenotyping and cytogenetic and molecular genetic analysis, have moved rapidly from being research tools to being sources of information which have an influence on patient management. Although rapid development is continuing, it seems an appropriate time to review the role of the various techniques now available from the viewpoint of haematologists working outside specialist leukaemia centres.

There are four main reasons for the thorough investigation of haematological malignancies: (i) to determine prognosis and to aid the selection of the best treatment for an individual; (ii) to advance scientific knowledge; (iii) to aid the education of trainees and the continuing education of haematologists; and (iv) to maintain the expertise and the interest of laboratory scientists. In the routine diagnostic laboratory the first reason is by far the most important but the significance of the others should not be underestimated. To confine investigations to those of immediate and obvious relevance to patient management would lead to the impoverishment of haematology and would ultimately lessen the quality of patient care. In addition, many non-specialist centres enter patients into clinical trials and in this context detailed investigation and accurate categorisation is important.

Although the development of immunophenotyping and of cytogenetic and molecular genetic analysis has lessened the importance of cytochemistry, the role of microscopy is undiminished. Careful examination of both peripheral blood and bone marrow films sometimes permits a definitive diagnosis and, when it does not, it at least provides a provisional diagnosis, indicates which of the more specialised investigations are most likely to be useful and provides a context for the interpretation of such specialised tests. Errors are likely to be made if microscopy is neglectedfor example, occasional cases of acute myeloid leukaemia (AML) show aberrant expression of a lymphoid antigen but fail to express any of the common myeloid antigens.

In this review I will not discuss details of techniques since up to date recommendations from expert groups are available. ${ }^{1-4}$ The role of different types of investigation will be discussed in relation to the provisional diagnosis, dealing separately with the acute leukaemias, myelodysplastic syndromes, chronic myeloid leukaemias, and chronic lymphoproliferative disorders. I shall use the French-American-British (FAB) terminology and classifications..$^{5-7}$

\section{The acute leukaemias}

The role of laboratory investigation in acute leukaemia is (i) to confirm the diagnosis of acute leukaemia, (ii) to distinguish AML from acute lymphoblastic leukaemia (ALL) and (iii) to identify certain subtypes of acute leukaemia which require special management. At present, it is important to identify acute promyelocytic leukaemia and its variant (M3 and M3V AML) and Burkitt type ALL (L3 ALL). The identification of M3/M3V AML is important because of the need to correct the associated haemostatic abnormality and because of the demonstrated efficacy of all-trans-retinoic acid in treatment. The identification of L3 ALL is important because of the greatly improved prognosis with appropriate intensive combination chemotherapy. ${ }^{89}$ It can also be important to identify certain cytogenetic subtypes of acute leukaemia which have such a poor prognosis with standard treatment that the use of "innovative and experimental therapy" is justified. Such highly unfavourable categories include Philadelphia $(\mathrm{Ph})$ positive acute leukaemia and infant ALL associated with karyotypic abnormalities having an 11q23 breakpoint.

\section{CYTOLOGY, HISTOLOGY AND CYTOCHEMISTRY}

Cytological assessment is of major importance in the diagnosis of acute leukaemia and, together with a limited range of cytochemical reactions, permits the diagnosis of the majority of cases of AML and a provisional diagnosis of ALL. Histological assessment of a trephine biopsy specimen is necessary for the diagnosis of hypocellular AML and for the diagnosis of those cases of M7 AML in which fibrosis prevents an adequate bone marrow aspirate being obtained. Histology is only necessary in ALL in the rare cases with a failed bone marrow aspirate.

The most important cytochemical tests are the myeloperoxidase (MPO) reaction or Sudan black B (SBB) stain for the identification of myeloid differentiation and a "non-specific" esterase reaction, such as $\alpha$-naphthyl acetate esterase (ANAE), for identification of monocytic differentiation. ${ }^{21}$ The MPO reaction permits the FAB categories of M1, M2, M3, M3V, 
and M4 AML to be identified as myeloid; many, but not all, cases of M5 AML are also MPO positive. A naphthol AS-D chloroacetate esterase (CAE) reaction is granulocyte specific but is less sensitive than MPO and SBB in the detection of myeloid differentiation. It should therefore not replace MPO or SBB although it is useful in a combined esterase reaction and occasional cases which are MPO and SBB negative are CAE positive.

A strong diffuse ANAE reaction permits identification of the monocytic component in M4 and M5 AML; however, in a minority of cases of M5a AML the non-specific esterase reaction is negative. ${ }^{12} \mathrm{~A}$ focal positive reaction is also obtained in some cases of M7 AML, in some $T$ lineage ALL and sometimes in the erythroblasts of M6 AML. The $\alpha$-naphthyl butyrate esterase reaction is an alternative to ANAE. It gives similar reaction patterns but is more specific for the monocyte lineage as megakaryoblasts give weak or negative reactions.

If mast cell or basophil differentiation is suspected, a toluidine blue stain is indicated, although metachromatic staining has also been reported in some cases of M3 AML. ${ }^{13}$ Primitive cells of basophil lineage are sometimes MPO and SBB positive but are CAE negative. Mast cells are CAE positive and SBB and MPO negative. They can be specifically identified by naphthol AS aminocaproate esterase positivity. ${ }^{14}$ Diagnosis of these rare leukaemias can be of some significance because of the clinical effects of secretion of histamine and other vasoactive substances.

The availability of immunophenotyping has greatly reduced the need for the periodic acidSchiff (PAS) and acid phosphatase reactions. Although the demonstration of PAS block positivity supports a diagnosis of ALL and focal acid phosphatase positivity supports a diagnosis of T lineage ALL, neither reaction is specific. ${ }^{11}$ These tests are therefore only necessary in circumstances when there is no access to immunophenotyping.

Cytochemical tests have been found to have some prognostic significance in acute leukaemia. PAS block positivity correlates with better prognosis in childhood $A L^{15}$ and SBB positivity with better prognosis in AML. ${ }^{16} \mathrm{How}-$ ever, in neither case is the prognostic significance of sufficient magnitude to influence patient management.

\section{IMMUNOPHENOTYPING}

Immunophenotyping is essential (i) to make a positive diagnosis of ALL and MO and M7 AML, (ii) to confirm the diagnosis of M5a AML when relevant cytochemical reactions are negative and (iii) to identify cases of AML in which the leukaemic cells are very primitive erythroid cells. Large cell lymphoma in leukaemic phase and small cell tumours of childhood can be confused with acute leukaemia if immunophenotyping is not used. Immunophenotyping is therefore indicated in all cases of suspected acute leukaemia which are not otherwise demonstrated to be myeloid.
In both ALL and AML, immunophenotyping can give information of relevance to prognosis and possibly to choice of treatment. This is so when mature B cell ALL is identified. Not all cases of B-ALL have L3 morphology and rare cases with L3 morphology are not BALL; the detection of membrane immunoglobulin is therefore important in confirming the diagnosis of L3/B-ALL and in identifying other cases of mature B-ALL which, although morphologically atypical, may nevertheless have Burkitt's lymphoma associated translocations ${ }^{17}$ and should be treated intensively. The presence of cytoplasmic $\mu$-chain (pre-BALL) also correlates with a worse prognosis in some, ${ }^{18}$ but not all, ${ }^{19}$ series but only because of its common association with $t(1 ; 19)$ (q23; p13). Although tests for cytoplasmic $\mu$-chain are now being more widely performed this test is not essential if cytogenetic analysis is performed in all cases. In some North American and European centres cases identified as $\mathrm{T}$ lineage ALL are treated more intensively or with alternative chemotherapy but in Britain this is not usually so. Among cases of AML there is evidence that CD7 positivity and CD34 positivity are adverse prognostic indicators but, at present, immunophenotyping is not of importance in determining choice of treatment. If the suspected poor prognosis of biphenotypic leukaemia is confirmed and alternative treatment for these cases is developed, the general use of immunophenotyping in all cases of acute leukaemia will become more important.

\section{CYTOGENETIC AND MOLECULAR GENETIC} ANALYSIS

Cytogenetic analysis in AML can be used to confirm a diagnosis and to predict prognosis and thus enable one to choose treatment which is related to the prognosis. Ideally, cytogenetic analysis should be carried out on all cases of acute leukaemia.

An example of the use of cytogenetics to confirm a diagnosis is the demonstration of the $t(15 ; 17)(q 22 ; q 11-12)$ in M3/M3V AML. There is rarely any difficulty in recognising classic M3 AML but M3V sometimes has aberrant cytochemical reactions and can be confused with acute monocytic leukaemia and with biphenotypic leukaemia with myeloid and natural killer cell markers. ${ }^{20}$ Cytogenetic analysis can also confirm the diagnosis in those cases of AML associated with $t(8 ; 21)(q 22 ; q 22)$ in which the blast percentage is unusually low.

Patients with AML can be divided into three prognostic groups on the basis of cytogenetic analysis. The most favourable cytogenetic categories are $t(8 ; 21)(\mathrm{q} 22 ; \mathrm{q} 22), \mathrm{t}(15 ; 17)(\mathrm{q} 22$; q11-12) and inv(16)(p13 q22). The most unfavourable are $t(9 ; 22)(\mathrm{q} 34 ; \mathrm{q} 11), \mathrm{t}(6 ; 9)(\mathrm{p} 23$; q34), inv(3)(q21 q26), complex karyotypic abnormalities, and deletions and monosomies of chromosomes 5 and 7. Trisomy 8 falls into an intermediate group. Although it is possible to define prognostic categories it is not yet clear to what extent the selective application of intensive therapy to adverse categories will improve the prognosis. However, radical therapy 
is clearly justifiable in young patients when the prognosis is otherwise very poor.

Karyotypic analysis is rarely needed to confirm the diagnosis of ALL but it is of some importance in identifying good and poor prognostic groups. ${ }^{21-23}$ Prognosis can only be considered in relation to treatment. With conventional, moderately intensive treatment a good prognosis has been associated with high hyperdiploidy (51 to 65 chromosomes) and with $\operatorname{dic}(9 ; 12)(\mathrm{p} 11 ; \mathrm{p} 12)$. A poor prognosis has been associated with the three Burkitt's lymphoma associated translocations and with near haploidy, hypodiploidy, $\mathrm{t}(4 ; 11)(\mathrm{q} 21 ; \mathrm{q} 23)$, and $t(9 ; 22)$ (q34; $\mathrm{q} 11)$. Intermediate prognosis is associated with del(6)(q). The prognosis of ALL with $\mathrm{t}(1 ; 19)$ (q23;p13) has improved when the presence of a translocation has been one of the factors which might lead to the selective application of more intensive treatment. ${ }^{22}$ The prognosis of $A L L$ with $t(9 ; 22)$ (q34;q11) or $t(4$; 11) (q21;q23) is so poor that very intensive treatment is clearly justifiable. It appears that at least for $\mathrm{Ph}$ positive ALL, results are better with bone marrow transplantation ${ }^{24}$ or alternative chemotherapy ${ }^{25}$ than with standard chemotherapy.

Cytogenetic analysis is indicated whenever treatment induced leukaemia is suspected, in order that such iatrogenic disorders should be recognised and documented. In addition to the well recognised karyotypic abnormalities associated with AML caused by alkylating agents and related drugs, a different range of abnormalities has more recently been recognised following the use of topoisomerase II interactive drugs. ${ }^{26}$ The latter group of drugs induces occasional cases of ALL as well as, more commonly, AML.

Karyotypic information of prognostic relevance can be obtained not only from standard cytogenetic analysis but also from newer techniques such as fluorescence in situ hybridisation (FISH), the DNA index and analysis of DNA or RNA (molecular genetic analysis). These techniques are useful when a few specific abnormalities are being sought-for example, in ALL, the BCR/ABL fusion gene associated with $\mathrm{t}(9 ; 22)$, the E2A/PBX1 fusion gene associated with $t(1 ; 19)$, and rearrangement of the MLL (HRX) gene associated with 11q23 breakpoints as in infants with $t(4 ; 11) .{ }^{10}$ In these situations molecular genetic analysis may be superior to cytogenetic analysis as adequate metaphases for analysis are not obtained in all cases of acute leukaemia and some cases without the usual translocation nevertheless have HRX or BCR/ABL rearrangement. In $\mathrm{ALL}$ an alternate technique to detect hyperdiploidy is the DNA index, determined by flow cytometry. This is an important prognostic indicator ${ }^{27}$ which is already being used for stratification in some trials.

\section{The myelodysplastic syndromes}

The reasons for performing specialised investigations in the myelodysplastic syndromes (MDS) are: (i) to confirm the diagnosis; and (ii) to determine prognosis and identify groups which may benefit from specific forms of treatment.

CYTOLOGY, CYTOCHEMISTRY AND HISTOLOGY Cytology of peripheral blood and bone marrow are crucial in suspected MDS and are often sufficient to establish the diagnosis. Cytochemistry is also important. A Perls' stain for haemosiderin and either a MPO or SBB stain is indicated. A Perls' stain permits the identification of ring sideroblasts, giving support for a diagnosis of MDS if there is no reason to suspect sideroblastic erythropoiesis secondary to drugs, chemicals or another bone marrow disease. Iron stores can also be assessed but a serum ferritin assay is a more reliable way to demonstrate iron overload. A MPO or SBB stain is indicated to ensure that any Auer rods present are identified as cases with Auer rods are more likely to respond favourably to chemotherapy. ${ }^{28}$ Cytochemical stains may give support for a diagnosis of MDS when maturing cells with enzyme deficiency or aberrant cytochemical reactions are identified. However, they are not often used for this purpose nor is the identification of the lineage of any blasts important as lymphoblastic transformation is uncommon and distinguishing between myeloblasts, megakaryoblasts and monoblasts has no therapeutic relevance.

A trephine biopsy is important whenever a poor bone marrow aspirate is obtained. Histological examination permits MDS to be distinguished from hypocellular AML and from aplastic anaemia. This diagnostic problem is particularly likely in secondary MDS which more often has a hypocellular bone marrow and bone marrow fibrosis. Histology is useful for identifying dyserythropoiesis and dysplastic megakaryocytes but is not very useful for the detection of dysgranulopoiesis. It will occasionally confirm a diagnosis of MDS, if abnormal localisation of immature precursors (ALIP) is detected. However, cases with ALIP usually have other features indicative of MDS in either peripheral blood or the bone marrow aspirate. Whether ALIP is an independent prognostic indicator is disputed, findings differing in different studies.

\section{IMMUNOPHENOTYPING}

Immunophenotyping can be used to assign a lineage to blast cells. However, this is of little therapeutic relevance and immunophenotyping is therefore not of any great importance in MDS.

\section{CYTOGENETIC AND MOLECULAR GENETIC}

ANALYSIS

Cytogenetic analysis can be used to confirm a diagnosis of MDS, particularly in cases with isolated cytopenia and minimal morphological evidence of dysplasia. In cases with characteristic cytological abnormalities karyotypic analysis is not necessary for diagnosis and the absence of a detectable clonal abnormality does not exclude MDS. 
The presence and nature of any cytogenetic abnormalities are also relevant to prognosis. Those abnormalities which are associated with a poor prognosis in AML are predictive, in MDS, of early leukaemic transformation and early death from the complications of cytopenia, whereas the presence of a normal karyotype or of $5 \mathrm{q}-$ or $20 \mathrm{q}$ - as an isolated abnormality is associated with a relatively good prognosis. Choice of treatment can be influenced by prognostic information derived from cytogenetic analysis. Thus iron chelation therapy might be indicated in a patient with refractory anaemia with $5 \mathrm{q}-$ as a sole abnormality whereas such therapy would have little relevance in a patient with $\operatorname{inv}(3)(\mathrm{q} 21 \mathrm{q} 26)$ or $\mathrm{t}(6 ; 9)(\mathrm{p} 23 ; \mathrm{q} 34)$ in whom early transformation and death would be likely. Conversely, bone marrow transplantation or treatment of the type used in acute leukaemia would be more relevant in a young patient with a very adverse karyotype.

Molecular genetic analysis is occasionally useful to demonstrate clonality and thus give support to a diagnosis of MDS. Otherwise, it does not have a major role in patient management.

\section{Chronic myeloid leukaemias}

Chronic myeloid leukaemia in adults can be further categorised as chronic granulocytic leukaemia (CGL), atypical chronic myeloid leukaemia (atypical CML) and chronic myelomonocytic leukaemia (CMML). Children may have juvenile $\mathrm{CML}$ and various myelodysplastic/myeloproliferative disorders, some of which are associated with the presence of monosomy 7. In general, the most useful diagnostic methods are examination of peripheral blood and cytogenetic and molecular genetic analysis.

CYTOLOGY, CYTOCHEMISTRY AND HISTOLOGY Peripheral blood cytology and leucocyte differential counts are crucial in the diagnosis of the CMLs. Ph positive CGL can be diagnosed with a high degree of reliability from the differential count alone.$^{29} \mathrm{Ph}$ negative CGL is haematologically identical. A diagnosis of atypical CML is likely in cases with immature granulocytes but with more prominent monocytosis than occurs in CGL; other atypical features which suggest this diagnosis are a greater degree of anaemia and thrombocytopenia and less consistent basophilia and eosinophilia. Atypical CML differs from CMML in that there are usually at least $5-15 \%$ of circulating immature granulocytic cells (blast cells to myelocytes). Cases of CMML do not often have more than 5\% immature granulocytes in the peripheral blood and rarely have eosinophilia or basophilia. Children very rarely develop CGL. Juvenile CML is a quite distinct disorder characterised by neutrophilia, monocytosis, anaemia, thrombocytopenia, and sometimes immature granulocytes and nucleated red blood cells in the peripheral blood; it also shows features typical of fetal erythropoiesis such as an elevated percentage of haemoglobin $\mathrm{F}$ and strongly expressed $\mathrm{i}$ antigen. Children may also develop a condition with some similarities to adult CMML, commonly associated with monosomy 7 .

In the CMLs examination of bone marrow is usually less informative than examination of peripheral blood, although it is necessary to exclude acute transformation and sometimes to distinguish atypical CML from AML.

Cytochemistry is no longer important in the diagnosis of CGL as, although the neutrophil alkaline phosphatase (NAP) score is lowered in about $95 \%$ of cases, cytogenetic and molecular genetic analysis permit a much more precise diagnosis. If these techniques are not available, the NAP score can be useful. A low NAP score favours a diagnosis of CGL but low scores are also seen in appreciable numbers of patients with other forms of CML. A reduced NAP score is common in atypical CML, although normal and even high scores are observed much more frequently than in CGL. Similarly, CMML may have either low or normal scores. The NAP score is more useful for distinguishing CGL from reactive conditions than from other myeloproliferative disorders. However, except in early cases with only a minor degree of elevation of the white cell count, there is rarely any difficulty in making this distinction.

Histological examination of a trephine biopsy specimen is not necessary for diagnosis.

\section{IMMUNOPHENOTYPING}

Immunophenotyping has no useful role during the chronic phase of the CMLs. CGL has a high probability of acute transformation which is lymphoblastic in about one third of cases. As remission is much more likely in lymphoblastic transformation, immunophenotyping is indicated whenever a transformation is not obviously myeloid. In myeloid transformations the precise lineage of the blasts is not of relevance to treatment and immunophenotyping is therefore not necessary. Blast transformation in the other CMLs is rarely lymphoid so that typing of blasts present during acute transformation is not necessary for patient management.

\section{CYTOGENETIC AND MOLECULAR GENETIC}

ANALYSIS

Karyotypic analysis is of considerable importance in the CMLs for making a precise diagnosis, for determining prognosis and for monitoring the response to treatment. Cytogenetic analysis is indicated in all cases but particularly in those patients in whom interferon therapy or bone marrow transplantation is being considered. The presence at diagnosis of other karyotypic abnormalities in addition to $\mathrm{t}(9 ; 22)(\mathrm{q} 34 ; \mathrm{q} 11)$ correlates with a worse prognosis ${ }^{30}$ and may influence choice of treatment. For this reason, it may be inappropriate for the detection of BCR/ABL rearrangement by molecular genetic analysis to replace cytogenetic analysis. However, molecular genetic analysis is indicated in $\mathrm{Ph}$ neg- 
ative cases, in order to confirm the diagnosis of $\mathrm{Ph}$ negative CGL. Identification of this condition is important as it appears to have the same biological characteristics and treatment response-for example, to interferon, as $\mathrm{Ph}$ positive CGL. ${ }^{3132}$

Karyotypic analysis is also sometimes necessary to distinguish between atypical CML and cases of CGL in which transformation has occurred before development of all of the features of chronic phase disease. The latter may have residual cytogenetically normal cells and further clonal abnormalities in addition to $t(9$; 22)(q34;q11).

Sequential cytogenetic analysis is indicated during therapy with interferon as the appearance of extra cytogenetic abnormalities may indicate a worse prognosis ${ }^{33}$ and as improved survival can be predicted by reversion to a $\mathrm{Ph}$ negative status or other major cytogenetic responses. ${ }^{3435}$ Cytogenetic monitoring is also indicated following bone marrow transplantation.

Cytogenetic analysis is indicated in childhood myeloproliferative/myelodysplastic conditions. The karyotype is often normal in juvenile CML, whereas monosomy 7 is common in other cases which lack the characteristics of fetal haemopoiesis.

\section{Other myeloproliferative disorders}

CYCTOLOGY, HISTOLOGY AND CYTOCHEMISTRY Examining the peripheral blood is important for diagnosing myeloproliferative disorders (MPD). In idiopathic myelofibrosis the characteristic blood film is very important for diagnosis. In other MPDs the detection of an increased basophil count, giant platelets or circulating megakaryocytes supports a diagnosis of MPD rather than a reactive condition. Bone marrow aspirates and trephine biopsy specimens are essential for the diagnosis of idiopathic myelofibrosis and systemic mastocytosis and are often useful in the diagnosis of the other MPDs if there is marked hypercellularity or abnormal megakaryocyte morphology or topography.

Cytochemistry is of minor importance in the diagnosis of MPDs other than CGL. The NAP score may be elevated in both polycythaemia rubra vera and essential thrombocythaemia and gives some support for the diagnosis of MPD; however, some patients with secondary polycythaemia and reactive thrombocytosis would also be expected to have an elevated NAP score. It is important to differentiate $\mathrm{Ph}$ positive essential thrombocythaemia, which has a considerably worse prognosis, from other cases of essential thrombocythaemia. It might be anticipated that the NAP score would be low in such cases and would help in making the distinction but this has been found not to be so. ${ }^{36}$

As acute transformation in MPDs other than CGL is almost always myeloid, cytochemistry for determining blast cell lineage is not clinically useful.
IMMUNOPHENOTYPING

Immunophenotyping is not generally useful in MPDs.

\section{CYTOGENETIC AND MOLECULAR GENETIC}

ANALYSIS

Cytogenetic analysis can be of use when a MPD is suspected but there is insufficient evidence to establish the diagnosis. The presence of a clonal cytogenetic abnormality is then confirmatory but its absence does not exclude the diagnosis. It is indicated in cases of "idiopathic hypereosinophilic syndrome" as some such cases have clonal cytogenetic abnormalities and are best regarded as having a MPD, specifically chronic eosinophilic leukaemia.

Cytogenetic analysis can give information relevant to prognosis. In essential thrombocythaemia not only the $\mathrm{Ph}$ chromosome but also inv(3)(q21q26) indicates a much worse prognosis and is likely to influence patient management. Cytogenetic analysis should therefore be carried out at least in cases with any atypical features-for example, blast cells in peripheral blood, a marked increase of the basophil count or dysplastic megakaryocytes. In idiopathic myelofibrosis the detection of any clonal cytogenetic abnormality indicates a worse prognosis in comparison with those in whom no such abnormality can be detected ${ }^{37}$ but as no effective treatment is available cytogenetic analysis is not generally clinically useful.

Molecular genetic analysis can be used to demonstrate BCR/ABL rearrangement in $\mathrm{Ph}$ positive essential thrombocythaemia and is occasionally useful for demonstrating clonality when the diagnosis of MPD is not certain.

\section{Chronic lymphoproliferative disorders}

The specific diagnosis of the various chronic lymphoproliferative disorders (LPD) is important as prognosis and optimal therapy differ. Cytology, histology and immunophenotyping have major and complementary roles both in the diagnosis and in the categorisation of LPD.

\section{CYTOLOGY, HISTOLOGY AND CYTOCHEMISTRY}

When peripheral blood and bone marrow are not involved in a LPD, diagnosis is based on histology supplemented, when necessary, by immunohistochemistry. When neoplastic cells are present in the peripheral blood or bone marrow, accurate diagnosis is often possible, without recourse to a lymph node biopsy, by means of an assessment of clinical, cytological and immunophenotypic features. In some conditions a diagnosis based on cytology and immunophenotyping is more likely to be accurate than one based on histology. This is true-for example, in large granular lymphocyte leukaemia, in T-prolymphocytic leukaemia (TPLL) and in adult T cell leukaemia/lymphoma. Cytological features of LPDs have been reviewed recently. ${ }^{38}$ Histology of a trephine biopsy specimen can also be very useful, particularly in distinguishing between chronic lymphocytic leukaemia (CLL) and non-Hodgkin's 
lymphoma, and in making a specific diagnosis of hairy cell leukaemia. The pattern of bone marrow infiltration in CLL may be interstitial, nodular or diffuse but is not paratrabecular, whereas in non-Hodgkin's lymphoma infiltration is often paratrabecular and rarely nodular. Hairy cell leukaemia shows a diffuse infiltrate with widely spaced nuclei with a fairly uniform chromatin pattern, which are surrounded by scanty ragged cytoplasm and an apparently empty space consequent on cell shrinkage. This appearance, which is more striking in paraffin wax than in plastic embedded sections, is so characteristic that it is sufficient to confirm the diagnosis.

Cytochemistry is of little importance in LPDs. Tartrate resistant acid phosphatase (TRAP) activity remains useful in the diagnosis of hairy cell leukaemia although it is not totally specific. Positive results are found in the great majority of cases of hairy cell leukaemia and in some cases of the variant form of hairy cell leukaemia. Positive reactions are sometimes seen in other LPDs-for example, in a minority of cases of B-prolymphocytic leukaemia and of splenic lymphoma with villous lymphocytes. The importance of the TRAP reaction has declined since the highly characteristic appearance of hairy cell leukaemia in trephine biopsy specimens has been appreciated and since the characteristic immunophenotypic profile ${ }^{39}$ has been defined. Nevertheless, laboratories which do not have the specific antibody panel necessary for an immunophenotypic diagnosis of hairy cell leukaemia may wish to test for TRAP positivity.

\section{IMMUNOPHENOTYPING}

Immunophenotyping is important in demonstrating the presence of a clonal population of cells, in differentiating chronic LPDs from ALL and in making a precise diagnosis of a LPD. Immunophenotyping is indicated in all cases of suspected LPD. It might be considered unnecessary in cases which appear to have typical clinical and cytological features of CLL, but in fact it is not uncommon for other conditions - for example, splenic lymphoma with villous lymphocytes, follicular lymphoma or the small cell variant of T-PLL, to be misdiagnosed as CLL if immunophenotyping is not performed.

Immunophenotyping is very useful in demonstrating light chain restriction in B-cell LPD, thus providing evidence of clonal proliferation. Some low grade non-Hodgkin's lymphomas, particularly mantle cell lymphoma, can be confused with reactive conditions on cytology alone and, conversely, post-splenectomy lymphocytosis and polyclonal $\mathrm{B}$ cell proliferation of cigarette smokers have been confused with neoplastic disorders until immunophenotyping was done. In $\mathrm{T}$ cell disorders immunophenotyping cannot establish clonality although a very uniform phenotype is suggestive.

Immunophenotyping is useful if there is any difficulty distinguishing between ALL and a chronic LPD. In ALL, with the exception of B-ALL, the neoplastic cells are terminal deoxy- nucleotidyl transferase ( $\mathrm{TdT}$ ) positive and do not express membrane immunoglobulin. $T$ and B cell lymphomas, with the exception of lymphoblastic lymphoma, are $\mathrm{TdT}$ negative and those of $\mathrm{B}$ lineage commonly express membrane immunoglobulin. The immunophenotype of B-ALL and Burkitt's lymphoma are the same; the cells express membrane immunoglobulin and are often, but not always, TdT negative.

CLL and hairy cell leukaemia have highly characteristic immunophenotypes ${ }^{339}$ which are virtually diagnostic. Other LPDs do not have such distinctive immunophenotypes but an assessment of both cytology and immunophenotype is often sufficient for a precise diagnosis. $^{339}$

\section{CYTOGENETIC AND MOLECULAR GENETIC} ANALYSIS

Certain karyotypic abnormalities are so strongly associated with specific lymphomas that cytogenetic analysis can confirm a provisional diagnosis. If there is no lymph node or other tissue available, cytogenetic analysis can be carried out on peripheral blood or bone marrow cells. The diagnosis of Burkitt's lymphoma, follicular lymphoma and mantle cell lymphoma can be confirmed in this manner. One of three specific translocations, $t(8 ; 14)$ (q24;q32), $\mathrm{t}(2 ; 8)(\mathrm{p} 11.2 ; \mathrm{q} 24)$ and $\mathrm{t}(8 ; 22)$ (q24;q11), is found in almost all cases of BALL and of Burkitt's lymphoma (endemic, sporadic and AIDS associated). Two variant translocations and $\mathrm{t}(14 ; 18)(\mathrm{q} 32 ; \mathrm{q} 21)$ are strongly associated with centroblastic/centrocytic (follicular) lymphoma, but are also found in some diffuse lymphomas when they indicate a follicular centre cell origin. The $t(11$; 14) (q13;q32) is found in about $50 \%$ of cases of mantle cell lymphoma, in about $20 \%$ of cases of splenic lymphoma with villous lymphocytes and in a smaller proportion of cases of Bprolymphocytic leukaemia. Among $\mathrm{T}$ lineage lymphomas, inv(14)(q11 q32) is strongly associated with T-PLL although the association is not totally specific. Demonstration of this cytogenetic abnormality is particularly useful in the diagnosis of the small cell variant of TPLL in which cytological diagnosis is sometimes difficult. Large cell anaplastic lymphoma of $\mathrm{T}$ lineage is strongly associated with $\mathrm{t}(2 ; 5)$ (p23;q35); this association can be diagnostically useful when histology is not straightforward-for example, when histiocytic proliferation and haemophagocytosis are very prominent.

The demonstration of a clonal karyotypic abnormality can be important, even when the abnormality found is not disease specific. In B cell disorders the demonstration of light chain restriction is a simpler method of demonstrating clonality, but in $\mathrm{T}$ cell disorders demonstrations of clonality rests on cytogenetic or molecular genetic analysis. Demonstration of a clonal cytogenetic abnormality gives strong support for a diagnosis of a LPD. (An exception is in ataxia telangiectasia in which an abnormal clone is sometimes present without any obvious 
signs of leukaemia or lymphoma.) Cytogenetic analysis can be particularly useful in cases with an excess of large granular lymphocytes as it can be difficult to distinguish, on the basis of clinical features and cytology, between reactive and neoplastic conditions. In CD3 negative cases without rearrangement of $\mathrm{T}$ cell receptor genes, demonstration of a karyotypic abnormality can be the only way to show that a proliferation is clonal and presumptively neoplastic.

Molecular genetic analysis can be used (i) to demonstrate clonality and (ii) to identify disease specific rearrangements. In B cell disorders clonality can be demonstrated by analysis of immunoglobulin heavy and light chain genes, while in $T$ cell disorders analysis of $T$ cell receptor genes gives similar information. If a condition is obviously neoplastic, demonstration of clonality is not necessary but it is useful in equivocal cases. DNA analysis is useful in $\mathrm{T}$ cell disorders and in $\mathrm{B}$ cell disorders which fail to express surface membrane or cytoplasmic immunoglobulin and which therefore cannot be studied for light chain restriction. Molecular genetic analysis can also be an alternative to cytogenetic analysis and can be used-for example, to demonstrate rearrangement of MYC and immunoglobulin genes in the Burkitt's lymphoma related translocations, rearrangement of $b c l-2$, as occurs in $\mathrm{t}(14 ; 18)(\mathrm{q} 32 ; \mathrm{q} 21)$ associated with follicular lymphoma, and rearrangement of $b c l-1$, as occurs in $t(11 ; 14)(\mathrm{q} 13 ; \mathrm{q} 32)$ associated with mantle cell lymphoma.

\section{Conclusions}

Traditional techniques of cytology, cytochemistry and histology used in the diagnosis of haematological neoplasms are now complemented by the techniques of immunophenotyping and cytogenetic and genetic analysis. Basic microscopy remains of vital importance and should not be neglected. Laboratories should also continue to perform a limited range of basic cytochemical reactions. More specialised tests are also often necessary for optimal patient management but laboratories need to decide whether such tests should be performed "in-house" or should be referred to a specialist centre. Such decisions are likely to be based on consideration of cost and on the speed with which tests results can be obtained from a specialist centre. This in turn is determined by geography, by staff levels and by the number of patients with suspected haematological neoplasms seen in a year. In making decisions based on cost-effectiveness laboratory managers should not forget the need to maintain the expertise and interest of technical and medical staff by providing continuing education and intellectual stimulation.

\section{Summary}

The relative importance of traditional techniques used in the diagnosis of haematological neoplasms has altered during the past decade. Cytology and histology retain their central role but the importance of cytochemistry has declined, except in the diagnosis of AML. Immunophenotyping is of major importance in the diagnosis of ALL, some categories of AML and the LPDs. Cytogenetic and molecular genetic analysis are important in the diagnosis of CML and are becoming increasingly important in the diagnosis of chronic LPDs and other haematological neoplasms. Diagnostic haematology laboratories which are not specialist leukaemia centres should have ready access to all of these techniques to ensure optimal patient management. However, not all techniques need to be performed in every laboratory.

$1 \mathrm{ICSH}$. Recommended procedures for cytological procedures in haematology. Clin Lab Haematol 1985;7:55-74.

2 ICSH. Recommended procedures for the classification of acute leukaemias. Leuk Lymph 1993;11:37-49.

3 General Haematology Task Force of BCSH. Immunophenotyping in the diagnosis of acute leukaemia. $\mathcal{f}$ Clin Pathol 1994;47:777-81.

4 General Haematology Task Force of BCSH. Immunophenotyping in the diagnosis of chronic lymphoproliferative disorders. $f$ Clin Pathol 1994;47:871-5.

5 Bennett JM, Catovsky D, Daniel MT, Flandrin G, Galton DAG, Gralnick HR, et al. Proposed revised criteria for the classification of acute myeloid leukemia. Ann Intern Med 1985;103:626-9.

6 Bennett JM, Catovsky D, Daniel M-T, Flandrin G, Galton DAG, Gralnick HR, et al. Proposals for the classification of chronic B and T lymphoid leukaemias. $\mathcal{f}$ Clin Pathol 1989;42:567-84.

7 Bennett JM, Catovsky D, Daniel MT, Flandrin G, Galton DAG, Gralnick HR, et al. Proposal for the recognition of minimally differentiated acute myeloid leukaemia (AMLMO). Br F Haematol 1991;78:325-9.

8 Murphy SB, Bowman WP, Abramowitch M, Mirro J, Ochs J, Rivera G, et al. Results of treatment of advanced stage Burkitt's lymphoma and B cell $(\mathbf{S I g}+)$ acute lymphoblastic leukemia with high-dose fractionated cyclophosphamide and coordinated high-dose methotrexate and cytarabine. and coordinated high-dose me

9 Hann IM, Eden OB, Barnes J, Pinkerton CR. "MACHO" chemotherapy for stage IV B lymphoma and B cell acute lymphoblastic leukaemia. Br F Haematol 1990;76:359-64.

10 Rubnitz JE, Link MP, Shuster JJ, Carroll AJ, Hakami N, Frankel LS, et al. Frequency and prognostic significance of HRX rearrangements in infant acute lymphoblastic leukaemia: a Pediatric Oncology Group study. Blood 1994; 84:570-3.

11 Bain B, Catovsky D. Current concerns in haematology 2 : Classification of acute leukaemia. F Clin Pathol 1990;43: 882-7.

12 Scott CS, Stark AN, Limbert HJ, Master PS, Head C, Roberts BE. Diagnostic and prognostic factors in acute Roberts BE. Diagnostic and prognostic factors in acute
monocytic leukaemia. Br $\mathcal{f}$ Haematol 1988;69:247-52.

13 Invernizzi R, Iannone AM, Bernuzzi S, D'Alessio A, Fiamenghi $\mathrm{C}$, Ippoliti $\mathrm{G}$, et al. Acute promyelocytic leukemia: morphological and clinical features. Haematologica 1993; 78:156-61.

14 Yam LT, Yam C-F, Li CY. Eosinophilia in systemic mastocytosis. Am 7 Clin Pathol 1980;73:48-54.

15 Lilleyman JS, Britton JA, Anderson LM, Richards SM, Bailey CC, Chessels JM on behalf of the Medical Research Bailey CC Chin Party on Childhood Leukaemia. Periodic Council Working Party on Childhood Leukaemia. Periodic acid Schiff reaction in childhood lymphoblastic leukaemia. f Clin Pathol 1994;47:689-92.

16 Hoyle CF, Gray RG, Wheatley K, Swirsky D, de Bastos M, Sherrington $\mathrm{P}$, et al. Prognostic importance of Sudan black positivity: a study of the bone marrow slides from 1386 patients with de novo acute myeloid leukaemia. $\mathrm{Br} \mathcal{F}$ Haematol 1991;79:398-407.

17 Traweek ST. Immunophenotypic analysis of acute leukemia Am f Clin Pathol 1993;99:504-12.

18 Crist WM, Carroll AJ, Shuster JJ, Behm FG, Whitehead $\mathrm{M}$, Vietti TJ, et al. Poor prognosis of children with pre-B acute lymphoblastic leukemia is associated with the $t(1$ 19)(q23;p13): a Pediatric Oncology Group Study, Blood 19)(q23;p13): a
1990;76:117-22.

19 Lilleyman JS, Hinchcliffe RF. Pre-B and "common" lymphoblastic leukaemia of childhood compared. $\mathrm{Br} f$ Haematol 1989;71:227-31.

20 Scott AA, Head DR, Kopecky K, Appelbaum FR, Theil KS, Grever MR, et al. HLA-DR-, CD 33+, CD56+, CD16 - myeloid/natural killer cell acute leukemia: a previously unrecognised form of acute leukemia potentially misdiagnosed as French-American-British acute myeloid leukemia-M3. Blood 1994;84:244-55.

21 Secker-Walker LM. Prognostic and biological importance of chromosome findings in acute lymphoblastic leukemia. Cancer Genet Cytogenet 1990;49:1-13.

22 Rivera GK, Raimondi SC, Hancock ML. Improved outcome 
in childhood acute lymphoblastic leukaemia with reinforced early treatment and rotating combination chemotherapy. Lancet 1991;337:61-6.

23 Raimondi SC. Current status of cytogenetic research in childhood acute lymphoblastic leukemia. Blood 1993;81: 2237-51.

24 Barrett AJ. Bone marrow transplantation for acute lymphoblastic leukaemia. Baillière's Clin Haematol 1994;7:377401.

25 Roberts WM, Rivera GK, Raimondi SC, Santana VM, Sandlund JT, Crist WM, et al. Intensive chemotherapy for Philadelphia-chromosome-positive acute lymphoblastic leukaemia. Lancet 1994;343:331-2.

26 Pederson-Bjergaard J, Rowley JD. The balanced and un balanced chromosome aberrations of acute myeloid leukemia may develop in different ways and may contribute differently to malignant transformation. Blood 1994;83: 2780-6.

27 Trueworthy R, Shuster J, Look T, Crist W, Borowitz M, Carroll A, et al. Ploidy of lymphoblasts is the strongest predictor of treatment outcome in B-progenitor cell acute lymphoblastic leukemia of childhood: a Pediatric Oncology Group study. F Clin Oncol 1992;10:606-13.

28 Seymour JF, Estey EH. The prognostic significance of Auer rods in myelodysplasia. Br $\mathcal{F}$ Haematol 1993;85:67-76.

29 Spiers ASD, Bain BJ, Turner J. The peripheral blood in chronic granulocytic leukaemia. Scand $\mathcal{f}$ Haematol 1977; 18:25-38.

30 Cervantes F, Robertson JE, Rozman C, Baccarani M, Tura $\mathrm{S}$, Gomez GA, et al. Long-term survivors in chronic granulocytic leukaemia: a study by the International CGL Prognosis Study Group. Br f Haematol 1994;87:293-300.
31 Shepherd PCA, Ganesan TS, Galton DAG. Haematological classification of the chronic myeloid leukaemias. Baillière Clin Haemat 1987;1:887-906.

32 Kantariian HM, Kurzrock R, Talpaz M. Philadelphia chromosome-negative chronic myelogenous leukemia and chronic myelomonocytic leukemia. Hematol Oncol Clin North Am 1990;4:389-404.

33 Lilleyman JS, Potter AM, Watmore AE, Cooke P, Sokol RJ, Wood JK. Myeloid karyotypes and the malignant phase of chronic granulocytic leukaemia. $\mathrm{Br} \mathcal{F}$ Haematol 1978; 39:317-23.

34 Morra E, Lazzarino M, Alimena G, Liberati AM, Grignani $\mathrm{F}$, Mandelli $\mathrm{F}$, et al. The role of interferon in the treatment of chronic myelogenous leukemia. Leuk Lymph 1992;6: of chronic

35 Kloke O, Niederle N, Qiu JY, Wandl U, Moritz T, NagelHiemke $\mathbf{M}$, et al. Impact of interferon alpha-induced cytogenetic improvement on survival in chronic myelogenous leukaemia. Br F Haematol 1993;83:399-403.

36 Stoll DR, Peterson P, Exten R, Laszlo J, Pisciotta AV, Ellis JT, et al. Clinical presentation and natural history of patients with essential thrombocythemia and the Phil adelphia chromosome. Am $\mathcal{f}$ Hematol 1988;27:77-83.

37 Demory $\pi$, Dupriez B, Fenaux $P$, Lai J, Beuscart $R$ Jouet JP, et al. Cytogenetic studies and their prognostic significance in chronic agnogenic myeloid metaplasia: a report of 47 cases. Blood 1988;72:855-9.

38 Bain BJ, Catovsky D. The leukaemic phase of non-Hodgkin's lymphoma. f Clin Pathol 1995;48:189-93.

39 Matutes E, Catovsky D. The value of scoring systems for the diagnosis of biphenotypic leukaemia and mature Bcell disorders. Leuk Lymph 1994;13(Suppl 1):11-14. 